\title{
Efficacy and Indications of Dexamethasone in Treatment of COVID-19
}

\begin{abstract}
Mikhail N* and Wali S
Endocrinology Division, Department of Medicine, Olive View-UCLA Medical Center, David-Geffen UCLA Medical School, CA, USA
\end{abstract}

*Corresponding author: Nasser Mikhail, Endocrinology Division, Department of Medicine, Olive View-UCLA Medical Center, David-Geffen UCLA Medical School, CA, USA, Tel: 7474-210-3205; Email: nmikhail@dhs. lacounty.gov

\section{Abstract}

Background: The precise role of glucocorticoids for treatment of coronavirus disease 2019 (COVID-19) is unclear. Objective: To review the therapeutic value of corticosteroids in hospitalized patients with COVID-19.

Methods: English literature search of electronic databases supplemented by manual search up to July 27, 2020. Search terms included glucocorticoids, corticosteroids, COVID-19, ARDS, dexamethasone, methylprednisolone, hydrocortisone, mortality, safety. Randomized trials, observational studies and major organization guidelines were included.

Results: Preliminary data from the only available large randomized trial in COVID-19, the "Randomized Evaluation of COVID-19 therapy" (RECOVERY) trial, showed that dexamethasone $6 \mathrm{mg} /$ day for up to 10 days reduced 28-day mortality (the primary outcome) in hospitalized patients with COVID-19 by $17 \%$, adjusted rate ratio (RR) $0.83,95 \%$ CI 0.75 to 0.93 (P < 0.001 ). The highest magnitude of mortality reduction was observed among patients receiving invasive mechanical ventilation, RR 0.64, 95\% CI; 0.51 to 0.81 ( $\mathrm{P}<0.001$ ), followed by patients receiving oxygen without mechanical ventilation, $\mathrm{RR} 0.82,95 \% \mathrm{CI}, 0.72$ to $0.94(\mathrm{P}=0.002)$. However, there was a non-significant trend toward increase mortality in patients not receiving respiratory support, RR 1.19, 95\% CI, 0.91 to 1.55. A smaller randomized study ( $\mathrm{n}=277)$ showed that dexamethasone decreased 60 -day mortality in mechanically ventilated patients with acute respiratory distress syndrome (ARDS), but without COVID-19. These latter results may be pertinent to COVID-19, in which ARDS is a common complication.

Conclusions: Dexamethasone $6 \mathrm{mg} / \mathrm{d}$ for up to 10 days should be included in treatment of patients with COVID-19 receiving invasive mechanical ventilation or oxygen. Meanwhile, dexamethasone should not be used in milder cases that do not require oxygen due to a possible harm.

Keywords: COVID-19; Dexamethasone; Glucocorticoids; Mortality; Mechanical ventilation; ARDS

\section{Introduction}

COVID-19 can elicit a severe inflammatory and pathological immune host response [1]. Therefore, use of corticosteroids as anti-inflammatory and immunosuppressive agents was used as part of treatment of COVID-19 [1]. Available data from small observational studies yielded conflicting results [2-4]. Meta-analyses may provide some insight. In the largest meta-analysis of 16 observational studies including 2,407 patients with
COVID-19 receiving corticosteroids, Zhang, et al. [5] reported that administration of corticosteroids was significantly associated with higher rates of ARDS $(\mathrm{P}<0.0003)$. On the other hand, a cohort Chinese study by $\mathrm{Wu}$, et al. [6] showed that patients with COVID-19 complicated by ARDS had lower mortality with methylprednisolone therapy (dose and duration not reported), Thus, mortality rates were 46.0\% (23 of 50 patients) and $61.8 \%$ (21 of 34 patients) in patients who received methylprednisolone treatment and those who did not receive it, respectively; hazard ratio 0.38 ; $05 \% \mathrm{CI}, 0.30$ to 


\section{Open Access Journal of Pulmonary \& Respiratory Sciences}

$0.72, \mathrm{P}=0.003$ ) [6]. Studies specifically using dexamethasone in COVID-19 are limited. In one series from the USA, Selvaraj, et al. [7] described 21 patients with COVID-19 and hypoxia admitted to the intermediate level of care unit. They received 6 day-course of dexamethasone $(4 \mathrm{mg}$ tid for 2 days, then $4 \mathrm{mg}$ bid for 2 days, then $4 \mathrm{mg}$ qd for 2 days) [7]. None of patients had escalation of care, and their C-reactive protein levels dropped by $79 \%$ on discharge [7]. Due to the observational non-randomized nature of the above studies, it is difficult to draw a conclusion regarding the therapeutic role of corticosteroids in COVID-19. Even when using a control group, there is inability to control for confounding factors (e.g. concomitant medications). Moreover, there is large variability in corticosteroid preparations, dosage, timing and duration of administration, and severity of the disease. Another major limitation inherent to these retrospective studies is indication bias, i.e. the sickest patients having the worst prognosis are those who are more likely to receive corticosteroids. Therefore, randomized trials are crucial to determine the status of corticosteroid therapy in COVID-19. The RECOVERY trial (see below) is the first randomized trial that examined the role of corticosteroids, specifically lowdose dexamethasone, for treatment of a large population $(n=6,425)$ of hospitalized patients with COVID-19 [8,9].

\section{Design of the RECOVERY Trial}

In the RECOVERY trial conducted in UK, the investigators randomized hospitalized patients with COVID-19 to 2 groups in a 2:1 ratio, the larger group $(n=4,321)$ received usual care while the smaller group $(n=2,104)$ received usual care plus low-dose of dexamethasone $6 \mathrm{mg} / \mathrm{d}$ orally or intravenously for up to 10 days in an open-label fashion [8]. The main results of the RECOVERY trial were announced in a preliminary form on June 16, 2020, just 98 days after the protocol was first drafted [8,9]. The primary outcome of RECOVERY is 28-day mortality [9]. Table 1 provides a summary of the design and main findings of the RECOVERY trial.

\begin{tabular}{|c|c|}
\hline Design & $\begin{array}{l}\text { Randomized, open-label, non-placebo controlled, } 2 \text { groups } \\
\text { 2:1 ratio, multi-center in UK }\end{array}$ \\
\hline Patients & $\begin{array}{l}\text { Hospitalized with COVID-19, mean age } 66.1 \text { years, } 36 \% \\
\text { woman, } 24 \% \text { with diabetes, } 27 \% \text { with heart disease }\end{array}$ \\
\hline Patient groups & $\begin{array}{l}\text { Usual standard care }(n=4,321) \text { versus standard care }+ \\
\text { dexamethasone }(n=2,104)\end{array}$ \\
\hline Dexamethasone regimen & $6 \mathrm{mg}$ once daily (oral or intravenously) for up to 10 days \\
\hline $\begin{array}{l}\text { Median duration of dexamethasone therapy (interquartile } \\
\text { range) }\end{array}$ & 7 days $(3$ to 10$)$ \\
\hline Primary outcome & 28-day mortality \\
\hline Secondary outcomes & $\begin{array}{l}\text { 1. Time to discharge, } 2 \text {. Subsequent receipt of mechanical } \\
\text { ventilation or death (composite outcome) }\end{array}$ \\
\hline Effect on dexamethasone on 26-day mortality & $\begin{array}{l}\text { Dexamethasone group } 482 \text { of } 2104(22.9 \%) \text {, usual care } \\
\text { group } 1110 \text { of } 4321(25.7 \%) \text {, *RR: } 0.83,95 \% \mathrm{CI}, 0.75 \text { to } \\
0.93 ; \mathrm{P}<0.001\end{array}$ \\
\hline $\begin{array}{l}\text { Effect of dexamethasone on } 28 \text {-day mortality among patients } \\
\text { on mechanical ventilation }(16 \% \text { of patients, } n=1,007)\end{array}$ & *RR; $0.64,95 \%$ CI 0.51 to 0.81 \\
\hline $\begin{array}{l}\text { Effect of dexamethasone on } 28 \text {-day mortality among patients } \\
\text { on oxygen only }(60 \% \text { of patients, } n=3,883)\end{array}$ & *RR: $0.82,95 \%$ CI 0.72 to 0.94 \\
\hline $\begin{array}{l}\text { Effect of dexamethasone on } 28 \text {-day mortality among patients } \\
\text { without respiratory support ( } 24 \% \text { of patients, } n=1,535)\end{array}$ & *RR: $1.19,95 \%$ CI 0.91 to 1.55 \\
\hline $\begin{array}{l}\text { Effect of dexamethasone on the first secondary outcome: } \\
\text { hospital discharge within } 28 \text { days }\end{array}$ & *RR: $1.11,95 \%$ CI, 1.03 to 1.17 \\
\hline $\begin{array}{l}\text { Effect of dexamethasone on the second secondary outcome: } \\
\text { subsequent receipt of mechanical ventilation or death }\end{array}$ & **RR: $0.92,95 \% \mathrm{CI}, 0.84$ to 1.01 \\
\hline
\end{tabular}

Table 1: Overview of the RECOVERY Trial [8].

${ }^{*} \mathrm{RR}=$ Rate ratio; ${ }^{* *} \mathrm{RR}=$ Risk ratio

\section{Primary Outcome of the RECOVERY Trial}

Significantly fewer patients assigned to dexamethasone reached the primary outcome of 28-day mortality compared with usual care, $22.9 \%$ (482 of 2104) and 25.7\% (1110 of 4321), respectively; RR 0.83; 95\% CI 0.74 to $0.93 ; \mathrm{P}<0.001$ ) [8]. Pre-specified subgroup analysis revealed significant trend of greatest absolute and proportional mortality reduction in the 


\section{Open Access Journal of Pulmonary \& Respiratory Sciences}

group of patients receiving invasive mechanical ventilation at randomization $(\mathrm{n}=1,007$ or $16 \%$ of study population) [8]. Thus, in the latter group of patients, dexamethasone was associated with mortality reduction of $36 \%$, RR 0.64 (95\% CI 0.51 to 0.81 ). In the subgroup of patients receiving oxygen ( $\mathrm{n}=3,883$ or $60 \%$ of study population), dexamethasone was associated with mortality reduction of $18 \%$, RR 0.82 , (95\% CI 0.72 to 0.94) [8]. Conversely, there was a nonsignificant trend toward an increase in 28-day mortality with dexamethasone in the subgroup of patients who were not receiving respiratory support $(\mathrm{n}=1,535$ or $24 \%$ of study population), RR 1.19 (95\% CI: 0.91 to 1.55) [8].

It should be emphasized that mortality rate in the usual care group, as expected, was highest among patients receiving mechanical ventilation reaching $40.7 \%$ [8]. It follows that the absolute mortality benefit of dexamethasone was also highest in this group of patients, 12.3 percentage points. In fact, based on these results, the authors estimated that 1 death would be prevented by dexamethasone treatment given to around 8 patients requiring invasive ventilation [9]. In patients receiving oxygen only, absolute mortality reduction was 4.1 percentage points [9]. Hence, in the latter group it is estimated that 1 death would be prevented by dexamethasone treatment of around 25 patients [9].

\section{Secondary Outcomes}

The RECOVERY trial included 2 secondary outcomes: duration of hospitalization, and a composite of receipt of invasive mechanical ventilation or death [8]. Thus, dexamethasone was associated with shorter duration of hospitalization than usual care, median 12 days and 13 days, respectively, and greater probability of discharge alive within 28 days (RR 1.10 (95\% CI, 1.03 to 1.17) [8]. Regarding the second secondary outcome, the number of patients progressing to mechanical ventilation or death was reduced among those allocated to dexamethasone compared with usual care, risk ratio being 0.92 (95\% CI 0.84 to 1.01 , $\mathrm{P}$ non-significant), and reduction was greater among patients receiving oxygen [8]. Risk of receipt or progression to invasive mechanical ventilation (a component of the second secondary outcome) was significantly reduced in the dexamethasone group risk ratio 0.77 ( $95 \%$ CI 0.62 to 0.95 ) [8].

\section{Subgroup Analysis}

Pre-specified subgroup analysis of the RECOVERY trial showed that dexamethasone was associated with reduction in 28-day mortality among patients with symptoms for more than 7 days but not among those with more recent symptom onset [8].

\section{Strengths of the RECOVERY Trial}

In general, the RECOVERY trial is a well-designed randomized controlled study with adequate statistical power including more than 6,000 patients with COVID-19 [8]. This trial provides answers to 3 important questions. First, who is the right patient with COVID-19 who benefit from treatment with glucocorticoids? Second, what type/dose of glucocorticoid? Third, what is the timing of administration of glucocorticoid?

Interestingly, no exclusion criteria were reported, except for patients known to have contraindications to dexamethasone [8]. Even pregnant and breast-feeding women and patients younger than 18 years-old were included $[8,9]$. Thus, this non-selected patient sample that represents $15 \%$ of all UK hospitalized patients makes results of the RECOVERY trial directly applicable to all hospitalized patients with COVID-19 worldwide. In fact, the greatest value of RECOVERY trial is that it is the first randomized trial to provide convincing high-quality evidence of mortality benefit among the sickest patients with COVID-19 [8]. Moreover, it is fortunate that the RECOVERY trial showed that this decrease in mortality was achieved by dexamethasone, a non-expensive and well-studied drug available since 1960s.

\section{Limitations of RECOVERY Trial}

The first limitation of the RECOVERY trial is its openlabel design and lack of a placebo group, and therefore may be open for bias [8]. While mortality is clearly a hard outcome, it cannot be excluded that investigator decision to withdraw mechanical ventilation might be influenced by type of assigned therapy. Second, the authors did not measure intermediate outcomes such as viral load and inflammatory markers that could clarify the mechanism of action of dexamethasone. Third, frequency of adverse effects of dexamethasone such as hyperglycemia, secondary infection, gastrointestinal bleeding, and psychosis were not reported. Fourth, minimal cross over of dexamethasone therapy occurred, with $8 \%$ of patients in the control group received dexamethasone [8]. However, the latter limitation should attenuate the mortality benefit of dexamethasone.

\section{Dexamethasone in Patients with Acute Respiratory Distress Syndrome without COVID-19}

Acute respiratory distress syndrome (ARDS) is a common complication of COVID-19 ranging from $41 \%$ among all patients admitted to the hospital to $71 \%$ of patients admitted to the intensive care units $[6,10]$. In the preliminary report from the RECOVERY trial, the proportion of patients 


\section{Open Access Journal of Pulmonary \& Respiratory Sciences}

with ARDS was not indicated. However, it is likely to be high, particularly in the group receiving mechanical ventilation. To the author's best knowledge, there is a single randomized controlled trial that evaluated corticosteroids, specifically dexamethasone, in patients with ARDS on mechanical ventilation but without COVID-19 [11]. In this Spanish study, one group of patients was allocated to dexamethasone + conventional treatment $(\mathrm{n}=139)$ and a second group of patients received conventional treatment alone $(n=138)$ [11]. The dexamethasone dose was 2.5 to 3 fold higher doses than in the RECOVERY trial, $20 \mathrm{mg}$ IV once daily for 5 days followed by $10 \mathrm{mg}$ IV once daily for the following 5 days [11]. The timing of initiation of dexamethasone was set to be within $30 \mathrm{~h}$ after confirmation of the diagnosis of ARDS [11]. The number of ventilator-free days at 28 days (the primary outcome) was significantly higher in the dexamethasone group compared with the conventional-treatment group, with a between-group difference of 4.8 days (95\% CI, 2.57 to 7.03; $\mathrm{P}<0.001$ ) [11]. Mortality at 60 days (the secondary outcome) was significantly lower in the dexamethasone group compared with control group, $21 \%$, and $36 \%$ respectively with a between-group absolute difference of $-15.3 \%$ (95\% CI, -25.9 to -4.9 ; $\mathrm{P}=0.0047$ ) [11]. The number needed to treat with dexamethasone to prevent one death in 60-day period was 7 patients [11]. Interestingly, these results are overall similar to those of the RECOVERY trial in the subgroup of COVID-19 patients receiving mechanical ventilation. Thus, in terms of mortality reduction, the absolute mortality difference at 28 days in the RECOVERY trial was 12.3 percentage points $(41.4 \%$ in control group versus $29.3 \%$ in dexamethasone group), and the number of patients needed to treat with dexamethasone to prevent one death at 28 days was 8 patients [9]. Although the pathophysiology of ARDS that complicates COVID-19 may not be identical to that associated with other diseases (e.g. pneumonia, sepsis, aspiration, trauma), the results of the above 2 trials taken together, suggest that dexamethasone may reduce mortality in mechanically ventilated patients having ARDS with and without COVID-19 $[8,11]$.

\section{Clinical Implications of the RECOVERY trial}

The RECOVERY trial is a breakthrough study with direct implications to clinical practice. The quality of data is sufficiently strong to implement the dexamethasone protocol used in the RECOVERY trial to all patients with COVID-19 receiving mechanical ventilation or oxygen. This implementation should be immediate due to the high mortality rates in patients with severe COVID-19, and absence of any other agent having a clear mortality benefit. Indeed, the RECOVERY protocol was adopted into UK practice on the same day the results were released $[8,9]$. Likewise, the National Institutes of Health in the USA updated its guidelines to recommend the same dexamethasone protocol of RECOVERY trial [12]. The World Health Organization (WHO) has welcomed the preliminary results of the RECOVERY trial [13]. However, up to the date of writing this manuscript (July 27,2020 ), the WHO is still in the process of updating treatment guidelines to include dexamethasone. It should be emphasized, however, that corticosteroids should not be used in patients with milder forms of COVID-19 who do not require oxygen as the results of RECOVERY trial suggest a possible harm.

\section{Conclusions and Current needs}

The RECOVERY trial has shown convincing evidence that dexamethasone in a low dose of $6 \mathrm{mg} / \mathrm{d}$ for up to 10 days significantly reduced mortality in patients with severe COVID-19 receiving invasive mechanical ventilation or oxygen, and among patients with symptoms for more than 7 days. These results are substantiated by a smaller randomized trial using higher doses of dexamethasone in non-COVID-19 patients with ARDS [11]. Meanwhile, the RECOVERY trial showed a trend towards increase mortality in patients with milder COVID-19 disease [8]. Several clinical trials are underway to further clarify the precise place of corticosteroids in treatment of COVID-19. These trials should further refine the optimum corticosteroid preparation, dosage, and timing of administration relative to the disease severity to achieve the maximal therapeutic benefit. Until such trials are available, the current evidence is sufficiently strong to follow the RECOVERY trial protocol of dexamethasone therapy in all patients with COVID-19 who require oxygen or mechanical ventilation.

\section{Conflict of Interest}

The authors do not have any conflict of interest to declare.

\section{References}

1. Zhang W, Zhao Y, Zhang F, Wang Q, Li T, et al. (2020) The use of anti-inflammatory drugs in the treatment of people with severe coronavirus disease 2019 (COVID-19): The perspectives of clinical immunologists from China. Clin Immunol 214: 108393.

2. Zhou W, Liu Y, Tian D, Wang C, Wang S, et al. (2020) Potential benefits of precise corticosteroids therapy for severe 2019-nCOV pneumonia. Signal Transduct Target Ther 5(1): 18.

3. Liu K, Fang YY, Deng Y, Wei L, Mei-Fang W, et al. (2020) Clinical characteristics of novel coronavirus cases in tertiary hospitals in Hubei province. Chin Med J 133(9): 


\section{Open Access Journal of Pulmonary \& Respiratory Sciences}

1025-1031.

4. Fadel R, Morrison AR, Vahia A, Smith ZR, Chaudhry Z, et al. (2020) Early short course corticosteroids in hospitalized patients with COVID-19. Clin Infect Dis.

5. Zhang JJY, Lee KS, Ang LW, Leo YS, Young BE, et al. (2020) Risk factors of severe disease and efficacy of treatment in patients with COVID-19: a systematic review, metaanalysis, and meta-regression analyis. Clin infect Dis.

6. Wu C, Chen X, Cai Y, Xia J, Zhou X, et al. (2020) Risk Factors Associated with Acute Respiratory Distress Syndrome and Death in Patients with Coronavirus Disease 2019 Pneumonia in Wuhan, China. JAMA Intern Med 180(7): 1-11.

7. Selvaraj V, Dappah-Afriyie K, Finn A, Flanigan TP (2013) Short-term dexamethasone in SARS-CoV-2 patients. R I Med J 103(6): 39-43.

8. The RECOVERY Collaborative Group (2020) Dexamethasone in hospitalized patients with Covid-19
- Preliminary Report. N Engl J Med.

9. Horby PW, Lim WS, Emberson J, Mafham M, Bell J, et al (2020) Effect of dexamethasone in hospitalized patients with COVID-19-Preliminary report. medRxiv.

10. Yu Y, Xu D, Fu S (2020) Patients with COVID-19 in 19 ICUs in Wuhan, China: a cross-sectional study. Crit Care 24: 219.

11. Villar J, Ferrando C, Martinez D, Ambros A, Munoz $\mathrm{T}$, et al. (2020) Dexamethasone treatment for the acute respiratory distress syndrome: a multicentre, randomised controlled trial. Lancet Respir Med 8(3): 267-276.

12. COVID-19 treatment guidelines. National Institutes of Health, Bethesda Softworks, USA.

13. WHO welcomes preliminary results about dexamethasone use in treating critically ill COVID-19 patients. 